PROCEEDINGS OF THE

AMERICAN MATHEMATICAL SOCIETY

Volume 140, Number 12, December 2012, Pages 4319-4322

S 0002-9939(2012)11255-9

Article electronically published on March 29, 2012

\title{
A NOTE ON THE ALMOST-SCHUR LEMMA ON 4-DIMENSIONAL RIEMANNIAN CLOSED MANIFOLDS
}

\author{
EZEQUIEL R. BARBOSA
}

(Communicated by Michael Wolf)

\begin{abstract}
In this short paper, we prove a type of the almost-Schur lemma, introduced by De Lellis-Topping, on 4-dimensional Riemannian closed manifolds assuming no conditions on the Ricci tensor or the scalar curvature.
\end{abstract}

\section{INTRODUCTION}

Let $(M, g)$ be an $\mathrm{n}(\geq 3)$-dimensinal closed Riemannian manifold. We say that $(M, g)$ is an Einstein manifold if its traceless Ricci tensor is identically zero:

$$
\text { Ric }_{g}-\frac{R_{g}}{n} g=0
$$

where $\operatorname{Ric}_{g}$ and $R_{g}$ denote the Ricci tensor and the scalar curvature, respectively, of the metric $g$. The Schur lemma states that every Einstein manifold of dimension $n \geq 3$ has constant scalar curvature. In [2, De Lellis and P. Topping ask to what extent the scalar curvature is constant if the traceless Ricci tensor is assumed to be small rather than identically zero. They proved the following result.

Theorem 1.1 (Almost-Schur Lemma [2]). Let $(M, g)$ be an $n(\geq 3)$-dimensinal closed Riemannian manifold with nonnegative Ricci tensor. Then

$$
\int_{M}\left|R i c_{g}-\frac{\bar{R}_{g}}{n} g\right|^{2} d v_{g} \leq \frac{n^{2}}{(n-2)^{2}} \int_{M}\left|R i c_{g}-\frac{R_{g}}{n} g\right|^{2} d v_{g},
$$

where $\bar{R}_{g}=\operatorname{vol}_{g}^{-1} \int_{M} R_{g} d v_{g}$ is the average of the scalar curvature $R_{g}$ of $g$.

In 2] they also showed that the constant in inequality (1.1) is optimal and the nonnegativity of the Ricci tensor cannot be removed in general: when $n \geq 5$ they gave examples of metrics on $\mathbb{S}^{n}$ which make the ratio of the left hand side of (1.1) to the right hand side of (1.1) arbitrarily large; when $n=3$, they found manifolds which makes the same ratio arbitrarily large. However, in the case of dimension $n=4$, they left it open. Then, Y. Ge and G. Wang, in 11, showed that Theorem 1.1 holds under the condition of nonnegativity of the scalar curvature for dimension $n=4$. They proved the following theorem.

Received by the editors October 13, 2010 and, in revised form, April 19, 2011 and May 13, 2011.

2010 Mathematics Subject Classification. Primary 53C25.

Key words and phrases. Einstein manifold, Schur's Theorem, 4-dimensional manifold.

The author was partially supported by CNPq-Brazil.

(C)2012 American Mathematical Society Reverts to public domain 28 years from publication 
Theorem 1.2 (Almost-Schur Lemma [2]). Let $(M, g)$ be a 4-dimensinal closed Riemannian manifold with nonnegative scalar curvature. Then

$$
\int_{M}\left|R i c_{g}-\frac{\bar{R}_{g}}{4} g\right|^{2} d v_{g} \leq 4 \int_{M}\left|R i c_{g}-\frac{R_{g}}{4} g\right|^{2} d v_{g},
$$

where $\bar{R}_{g}=\operatorname{vol}_{g}(M)^{-1} \int_{M} R_{g} d v_{g}$ is the average of the scalar curvature $R_{g}$ of $g$. Moreover, equality in (1.2) holds if and only if $(M, g)$ is an Einstein manifold.

In this note we will prove a type of the almost-Schur lemma in dimension $n=4$ assuming no conditions on the Ricci tensor or the scalar curvature. Our main result is the following.

Theorem 1.3. Let $(M, g)$ be a 4-dimensinal closed Riemannian manifold. Then

$$
\int_{M}\left|R i c_{g}-\frac{\bar{R}_{g}}{4} g\right|^{2} d v_{g} \leq 4 \int_{M}\left|R i c_{g}-\frac{R_{g}}{4} g\right|^{2} d v_{g}+9 \lambda_{g}^{2}-\frac{\bar{R}_{g}}{4} \int_{M} R_{g} d v_{g},
$$

where $\bar{R}_{g}=\operatorname{vol}_{g}(M)^{-1} \int_{M} R_{g} d v_{g}$ is the average of the scalar curvature $R_{g}$ of $g$ and $\lambda_{g}$ is the Yamabe invariant. Moreover, equality in (1.3) holds if and only if there exists a metric $g_{1} \in[g]$ such that $\left(M, g_{1}\right)$ is an Einstein manifold.

We will note that if the Yamabe invariant $\lambda_{g}$ is nonnegative, then

$$
9 \lambda_{g}^{2}-\frac{\bar{R}_{g}}{4} \int_{M} R_{g} d v_{g} \leq 0 .
$$

Hence, as an immediate consequence of Theorem 1.3 we obtain the following corollary.

Corollary 1.4. Let $(M, g)$ be a 4-dimensinal closed Riemannian manifold with nonnegative Yamabe invariant. Then

$$
\int_{M}\left|R i c_{g}-\frac{\bar{R}_{g}}{4} g\right|^{2} d v_{g} \leq 4 \int_{M}\left|R i c_{g}-\frac{R_{g}}{4} g\right|^{2} d v_{g},
$$

where $\bar{R}_{g}=$ vol $_{g}(M)^{-1} \int_{M} R_{g} d v_{g}$ is the average of the scalar curvature $R_{g}$ of $g$. Moreover, equality in (1.4) holds if and only if $(M, g)$ is an Einstein manifold.

\section{Proof of Theorem 1.3}

Proof. Let

$$
S_{g}=\frac{1}{n-2}\left(R i c_{g}-\frac{R_{g}}{2(n-1)} g\right)
$$

be the Schouten tensor of $g$. For an integer $k$ with $1 \leq k \leq n$ let $\sigma_{k}$ be the $k$-th elementary symmetric function in $\mathbb{R}^{n}$. The $k$-scalar curvature is

$$
\sigma_{k}(g):=\sigma_{k}\left(\Lambda_{g}\right),
$$

where $\Lambda_{g}$ is the set of eigenvalues of the matrix $g^{-1} \cdot S_{g}$. In particular, $\sigma_{1}(g)=\operatorname{tr} S_{g}$ and $\sigma_{2}=\frac{1}{2}\left(\left(t r S_{g}\right)^{2}-\left|S_{g}\right|^{2}\right)$. Here, the Yamabe invariant is defined by

$$
\lambda_{g}=\inf _{\bar{g} \in[g]} \frac{\int_{M} \sigma_{1}(\bar{g}) d v_{\bar{g}}}{v o l_{\bar{g}}(M)^{\frac{n-2}{n}}},
$$


where $[g]$ denotes the conformal class of the metric $g$. Note that the definition of the Yamabe invariant is different from the standard one by a multiple factor $\frac{1}{2(n-1)}$. We can find the following identities:

$$
\begin{gathered}
\sigma_{1}(g)=\frac{R_{g}}{2(n-1)}, \\
\sigma_{2}(g)=\frac{1}{2(n-2)^{2}}\left(-\left|R i c_{g}\right|^{2}+\frac{n}{4(n-1)} R_{g}^{2}\right), \\
\left|R i c_{g}-\frac{R_{g}}{4} g\right|^{2}=\left|R i c_{g}\right|^{2}-\frac{R_{g}^{2}}{4}
\end{gathered}
$$

and

$$
\left|R i c_{g}-\frac{\bar{R}_{g}}{4} g\right|^{2}=\left|R i c_{g}\right|^{2}-\frac{\bar{R}_{g}}{2} R_{g}+\frac{\bar{R}_{g}^{2}}{4} .
$$

Hence, we see that inequality (1.3) is equivalent to the following:

$$
\frac{8}{3} \int_{M} \sigma_{2}(g) d v_{g} \leq \lambda_{g}^{2} .
$$

The proof of (2.1) closely follows an argument given by Gursky in 3 and Ge-Wang in [1. Let $g_{1}$ be a solution of the Yamabe problem. Thus the scalar curvature, and hence $\sigma_{1}\left(g_{1}\right)$, is constant. First, we have that for any $4 \times 4$ symmetric matrix $A$, the

$$
\left(\sigma_{1}(A)\right)^{2} \geq \frac{8}{3} \sigma_{2}(A)
$$

equality holds if and only if the matrix is a multiple of the identity one. Now the following calculations lead to

$$
\frac{8}{3} \operatorname{vol}_{g_{1}}(M) \int_{M} \sigma_{2}\left(g_{1}\right) d v_{g} \leq \operatorname{vol}_{g_{1}} \int_{M}\left(\sigma_{1}\left(g_{1}\right)\right)^{2} d v_{g}=\left(\int_{M} \sigma_{1}\left(g_{1}\right) d v_{g}\right)^{2},
$$

since $\sigma_{1}\left(g_{1}\right)$ is a constant. Therefore,

$$
\frac{8}{3} \int_{M} \sigma_{2}\left(g_{1}\right) d v_{g_{1}} \leq\left(\frac{1}{\left(\operatorname{vol}_{g_{1}}(M)\right)^{\frac{1}{2}}} \int_{M} \sigma_{1}\left(g_{1}\right) d v_{g_{1}}\right)^{2}=\lambda_{g}^{2},
$$

since $g_{1}$ is a Yamabe solution. Now, in the case of dimension $n=4$, it is well-known that $\int_{M} \sigma_{2}(g) d v_{g}$ is constant in any given conformal class. Hence,

$$
\frac{8}{3} \int_{M} \sigma_{2}(g) d v_{g}=\frac{8}{3} \int_{M} \sigma_{2}\left(g_{1}\right) d v_{g_{1}} \leq \lambda_{g}^{2},
$$

and this proves the inequality (2.1). Finally, observe that if the equality holds in (1.3), then

$$
\frac{8}{3} \int_{M} \sigma_{2}\left(g_{1}\right) d v_{g_{1}}=\int_{M}\left(\sigma_{1}\left(g_{1}\right)\right)^{2} d v_{g_{1}}
$$

and the Schouten tensor $S_{g_{1}}$ is proportional to the metric $g_{1}$, i.e., $g_{1}$ is an Einstein metric. On the other hand, if there exists an Einstein metric $h \in[g]$, we find from [4] that each Yamabe metric $g_{1}$ is an Einstein metric. Hence, in this case we obtain that

$$
\frac{8}{3} \int_{M} \sigma_{2}\left(g_{1}\right) d v_{g_{1}}=\lambda_{g}^{2} .
$$


Hence,

$$
\frac{8}{3} \int_{M} \sigma_{2}(g) d v_{g}=\frac{8}{3} \int_{M} \sigma_{2}\left(g_{1}\right) d v_{g_{1}}=\lambda_{g}^{2},
$$

and the equality holds in (1.3).

\section{REFERENCES}

1. Y. Ge, G. Wang, An almost Schur Theorem on 4-dimensional manifolds, Proc. Amer. Math. Soc. 140 (2012), pp. 1041-1044.

2. C. De Lellis, P. Topping, Almost-Schur Lemma, to appear in Calc. Var. and PDE.

3. M. Gursky, The principal eigenvalue of a conformally invariant differential operator, with an application to semilinear elliptic PDE, Comm. Math. Phys. 207 (1999), pp. 131-143. MR.1724863 (2000k:58029)

4. M. Obata, The conjectures on conformal transformations of Riemannian manifolds, J. Diff. Geo. 6 (1971), pp. 247-258. MR.0303464 (46:2601)

Department of Mathematics, ICEx, Universidade Federal de Minas Gerais, C.P. 702, Belo Horizonte, MG, CEP 30161-970, Brazil

E-mail address: ezequiel@mat.ufmg.br 